\title{
Comparison Between the Exact Solutions of Three Distinct Shallow Water Equations Using the Painlevé Approach and Its Numerical Solutions
}

\author{
A. Bekir, M.S. M. Shehata, E. H. M. Zahran
}

In this article, we employ the Painlevé approach to realize the solitary wave solution to three distinct important equations for the shallow water derived from the generalized CamassaHolm equation with periodic boundary conditions. The first one is the Camassa-Holm equation, which is the main source for the shallow water waves without hydrostatic pressure that describes the unidirectional propagation of waves at the free surface of shallow water under the influence of gravity. While the second, the Novikov equation as a new integrable equation, possesses a bi-Hamiltonian structure and an infinite sequence of conserved quantities. Finally, the third equation is the $(3+1)$-dimensional Kadomtsev-Petviashvili (KP) equation. All the ansatz methods with their modifications, whether they satisfy the balance rule or not, fail to construct the exact and solitary solutions to the first two models. Furthermore, the numerical solutions to these three equations have been constructed using the variational iteration method.

Keywords: Camassa-Holm equation, Novikov-Veselov equation, (3+1)-dimensional Kadomtsev - Petviashvili (KP) equation, Painlevé approach, traveling wave solutions, numerical solutions

Received August 04, 2020

Accepted August 26, 2020

Ahmet Bekir

bekirahmet@gmail.com

Neighbourhood of Akcaglan, Imarli Street

Number: 28/4, 26030, Eskisehir, Turkey

Maha S. M. Shehata

dr.maha_32@hotmail.com

Zagazig University, Faculty of Science, Departments of Mathematics

44519, Zagazig, Egypt

Emad H. M. Zahran

e_h_zahran@hotmail.com

Benha University, Faculty of Engineering, Departments of Mathematical and Physical Engineering

Fareed Nada Street, 13511, Shubra, Egypt 


\section{Introduction}

The main idea of this paper concentrates on using the Painlevé approach for the first time to realize the solitary wave solution to these three important models of shallow water as well as demonstrating the corresponding numerical solutions according to the variational iteration method. Traveling wave solutions represent waves of permanent shape $Z$ that propagate at constant speed $C_{0}$. These waves are called solitary waves on the condition that the wave profile $Z$ decays at infinity. If the solitary waves retain their shape and speed after interacting with other waves of the same type, we say that the solitary waves are solitons. There is a close connection between integrability and solitons. The solitons become peaked if they are shaped like the graph of the function $Z(x)=e^{-|x|}$, and they are then called peakons. It is interesting to notice also the property of orbital stability for the solitary waves, i.e., their shape is stable under small perturbations, both for the smooth solitons and for the peakons. Independently, Fokas and Fuchssteiner [1, 2] derived the Camasss - Holm equation and studied completely the integrable generalizations of the KdV equation with bi-Hamiltonian structures. Also, in the same context Camassa and Holm [3] (see also [4]) derived it as a nonlinear wave equation in which the wavelength is larger compared with the average water depth and which models unidirectional wave propagation on shallow water, and discovered its rich mathematical structure. In this context, we employ the Camassa - Holm equation in terms of the function $u(x, t)$ which represents the fluid velocity in the $x$-direction at time $t$. This equation can be written in the form [5]

$$
u_{t}-u_{x x t}+2 k u_{x}+3 u u_{x}-2 u_{x} u_{x x}-u u_{x x x}=0,
$$

where $k$ is related to the critical wave speed $C_{0}=\sqrt{g H_{0}}$ where $H_{0}$ denotes the undivided depth. Specifically, we shall focus on the case $k=0$ which in fact is not limited since the change of variables $u=u(x, t)$ that represent the fluid velocity in the $x$-direction, $u(x, t)=v(x-k t, t)+k$, tends to zero at $k=0$. In this case the Camassa-Holm equation has peakon solutions, i.e., solitons with a sharp peak, so with a discontinuity at the peak in the wave slope. According to [6-8], the formalism which describes the unidirectional propagation of shallow water waves over a flat bottom can be represented as

$$
u_{t}-u_{x x t}+3 u u_{x}-2 u_{x} u_{x x}-u u_{x x x}=0 .
$$

Secondly, we will solve the Novikov - Veselov equation, which is a natural $(2+1)$-dimensional analogue of the Korteweg-de Vries (KdV) equation [9-11]. This equation was named in 2009 after S. P. Novikov and A. P. Veselov [12], who derived a new integrable Camassa-Holm type equation with cubic nonlinearity which possesses a bi-Hamiltonian structure and an infinite sequence of conserved quantities and admits exact peaked solutions in the form

$$
u_{t}-u_{x x t}+4 u^{2} u_{x}-3 u u_{x} u_{x x}-u^{2} u_{x x x}=0 .
$$

Some results on the existence and uniqueness of global weak solutions to the Novikov equation have been realized by $\mathrm{Wu}$ and Yin [13]. The periodic Novikov equation is locally well-posed and was proved by Tiglay [14]. In [15] the range of regularity index of local well-posedness was extended; furthermore, it is clear that there is incompatible continuity from any bounded subset between the maps solution to both the periodic boundary-value problem and the Cauchy problem of the Novikov equation. The character of solutions to the Novikov - Veselov equation depends basically on the regularity of the scattering data for this solution. If the scattering data have no singularity, then the solution disappears uniformly with time. If the scattering data 
are not regular, then the solution may develop solitons. Furthermore, at positive energy the solitons of the Novikov - Veselov equation are transparent potentials as in the one-dimensional case (in which solitons have reflection less than potentials). Also, the well-known exponentially decaying solitons are realized when the similarity of one-dimensional case is not satisfied.

Finally, we will solve the $(3+1)$-dimensional Kadomtsev-Petviashvili (KP) equation [16]

$$
u_{x t}+6 u_{x}^{2}+6 u u_{x x}-u_{x x x x}-u_{y y}-u_{z z}=0,
$$

which describes the dynamics of weakly dispersive, nonlinear waves whose wavelength is large compared to its amplitude and whose variations in the main direction of propagation (rescaled $X$ ) are fast compared to their variations in the second space dimension (rescaled $Y$ ). Examples of these phenomena include: (1) the surface waves in shallow water, in which $u$ is a rescaled wave amplitude and a rescaled velocity; the wavelength is long compared to the depth of the water $h$, which is large compared to the wave amplitude; (2) magneto-elastic waves in antiferromagnetic materials, in which $u$ is a rescaled strain tensor and a rescaled velocity.

\section{Description of the Painlevé approach}

To propose the general formalism of the nonlinear evolution equation, let us introduce $R$ as a function of $(x, t)$ and its partial derivatives as

$$
R\left(\varphi, \varphi_{x}, \varphi_{t}, \varphi_{x x}, \varphi_{t t}, \ldots\right)=0
$$

which involves the highest-order derivatives and nonlinear terms. With the aid of the transformation $\varphi(x, t)=\varphi(\zeta), \zeta=x-C_{0} t$ Eq. (2.1) can be reduced to the following ODE:

$$
S\left(\varphi^{\prime}, \varphi^{\prime \prime}, \varphi^{\prime \prime \prime}, \ldots\right)=0,
$$

where $S$ is a function in $\varphi(\zeta)$ and its total derivatives.

According to the Painlevé approach [17], the exact solution to the nonlinear ordinary differential equation can be written in the following form:

$$
\varphi(\zeta)=A_{0}+S(X) e^{-N \zeta}, \quad X=R(\zeta)
$$

or

$$
\varphi(\zeta)=A_{0}+A_{1} S(X) e^{-N \zeta}+A_{2} S^{2}(X) e^{-2 N \zeta}, \quad X=R(\zeta),
$$

where $X=R(\zeta)=C_{1}-\frac{e^{-N \zeta}}{N}$ and $S(X)$ in Eq. (2.3) satisfies the Riccati-equation in the form $S_{X}-A S^{2}=0$. One can solve this equation to get

$$
S(X)=\frac{1}{S X+X_{0}} .
$$

Consequently,

$$
\begin{gathered}
\varphi_{\zeta}=-N e^{-N \zeta} S(X)+R_{\zeta} e^{-N \zeta} S_{X} \\
\varphi_{\zeta \zeta}=N^{2} e^{-N \zeta} S(X)-2 N R_{\zeta} e^{-N \zeta} S_{X}+R_{\zeta \zeta} e^{-N \zeta} S_{X}+R_{\zeta}^{2} e^{-N \zeta} S_{X X}, \\
\varphi_{\zeta \zeta \zeta}=-N^{3} S(X) e^{-N \zeta}+3 N^{2} R_{\zeta} S_{X} e^{-N \zeta}-3 N R_{\zeta \zeta} S_{X} e^{-N \zeta}- \\
-3 N R_{\zeta}^{2} S_{X X} e^{-N \zeta}+3 R_{\zeta} R_{\zeta \zeta} S_{X X}+R_{\zeta \zeta \zeta} S_{X} e^{-N \zeta}+R_{\zeta}^{3} S_{X X X} e^{-N \zeta}
\end{gathered}
$$




\section{Application}

In this section, we will apply the Painlevé approach as a new technique to realize the exact solutions to the Camassa-Holm model, the Novikov - Veselov equations in terms of some variables. Hence, we can easily obtain the traveling wave solutions when these variables take specific values.

\subsection{The Camassa-Holm equation}

Firstly, we will apply the constructed approach to Eq. (1.2) mentioned above:

$$
u_{t}-u_{x x t}+3 u u_{x}-2 u_{x} u_{x x}-u u_{x x x}=0 .
$$

By admitting the transformation $u(x, t)=\varphi(\zeta), \zeta=x-C_{0} t$, we get

$$
-C_{0} \varphi_{\zeta}+C_{0} \varphi_{\zeta \zeta \zeta}+3 \varphi \varphi_{\zeta}-2 \varphi_{\zeta} \varphi_{\zeta \zeta}-\varphi \varphi_{\zeta \zeta \zeta}=0 .
$$

According to the proposed method, the suggested solution is

$$
\varphi(\zeta)=A_{0}+e^{-N \zeta} S(X), \quad X(\zeta)=R(\zeta)=C_{1}-\frac{e^{-N \zeta}}{N} .
$$

Thus, we can easily obtain

$$
\begin{aligned}
\varphi_{\zeta} & =-N e^{-N \zeta} S-A e^{-2 N \zeta} S^{2}, \\
\varphi_{\zeta \zeta} & =N^{2} e^{-N \zeta} S+3 A N e^{-2 N \zeta} S^{2}+2 A^{2} e^{-3 N \zeta} S^{3}, \\
\varphi_{\zeta \zeta \zeta} & =-N^{3} e^{-N \zeta} S-7 A N^{2} e^{-2 N \zeta} S^{2}-12 A^{2} N e^{-3 N \zeta} S^{3}-6 A^{3} e^{-4 N \zeta} S^{3} .
\end{aligned}
$$

Substituting $\varphi_{\zeta}, \varphi_{\zeta \zeta}$ and $\varphi_{\zeta \zeta \zeta}$ into Eq. (3.2) and equating the coefficients of different powers of $S(\zeta) e^{-N \zeta}$ to zero implies the following two cases:

(i) when

$$
N=1, \quad \text { and } \quad A=\frac{25}{16 C_{0}},
$$

the solution is

$$
\varphi(\zeta)=\frac{16 C_{0} e^{-\zeta}}{25 X+16 C_{0} X_{0}}
$$

(ii) when

$$
N=-1, \quad \text { and } \quad A=\frac{-1}{2 C_{0}},
$$

the solution is

$$
\varphi(\zeta)=\frac{-2 C_{0} e^{\zeta}}{X-2 C_{0} X_{0}} .
$$

\subsection{The Novikov - Veselov equation}

Secondly, we will apply the constructed approach to Eq. (1.3) mentioned above:

$$
u_{t}-u_{x x t}+4 u^{2} u_{x}-3 u u_{x} u_{x x}-u^{2} u_{x x x}=0 .
$$

By admitting the transformation $u(x, t)=\varphi(\zeta), \zeta=x-C_{0} t$, we get

$$
-C_{0} \varphi_{\zeta}+C_{0} \varphi_{\zeta \zeta \zeta}+4 \varphi \varphi_{\zeta}-2 \varphi \varphi_{\zeta} \varphi_{\zeta \zeta}-\varphi^{2} \varphi_{\zeta \zeta \zeta}=0 .
$$

Using the transformation (3.1), substituting $\varphi_{\zeta}, \varphi_{\zeta \zeta}$ and $\varphi_{\zeta \zeta \zeta}$ into Eq. (3.11) and equating the coefficients of different powers of $\varphi(\zeta)$ to zero, we obtain the following two cases: 

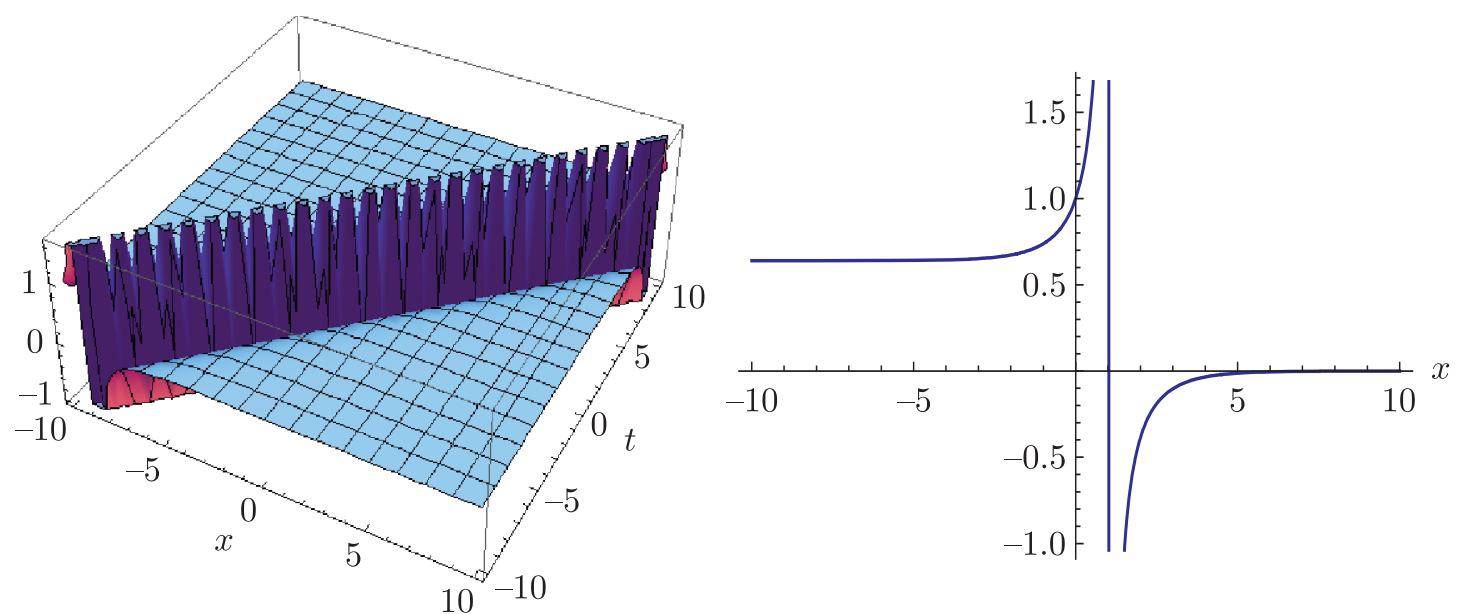

Fig. 1. The plot of Eq. (3.8) in $\mathbf{2 D}$ and $\mathbf{3 D}$ with values: $N=1, A=\frac{25}{16 C_{0}}, C_{0}=1, X_{0}=1, C_{1}=1$.
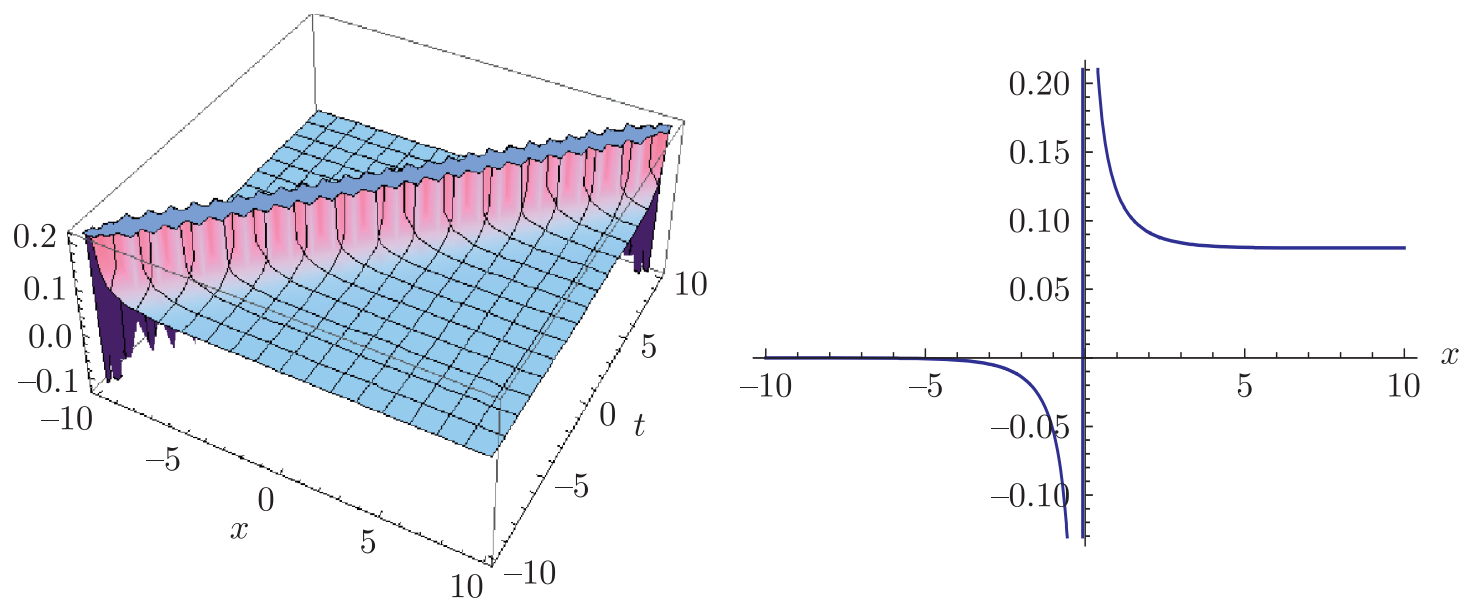

Fig. 2. The plot of Eq. (3.10) in $\mathbf{2 D}$ and $\mathbf{3 D}$ with values: $N=-1, A=\frac{-1}{2 C_{0}}, C_{0}=1, X_{0}=1, C_{1}=1$.

(i) when

$$
N=1, \quad \text { and } \quad A=\frac{-1-2 C_{0} \pm \sqrt{1+1174 C_{0}^{2}}}{9 C_{0}}
$$

the solution is

$$
\varphi(\zeta)=\frac{9 C_{0} e^{-\zeta}}{\left(-1-2 C_{0} \pm \sqrt{1+1174 C_{0}^{2}}\right) X+9 C_{0} X_{0}} ;
$$

(ii) when

$$
N=-1, \quad \text { and } \quad A=\frac{2-3 C_{0} \pm \sqrt{9 C_{0}^{2}-102 C_{0}+4}}{6 C_{0}}
$$

the solution is

$$
\varphi(\zeta)=\frac{6 C_{0} e^{\zeta}}{\left(2-3 C_{0} \pm \sqrt{9 C_{0}^{2}-102 C_{0}+4}\right) X+6 C_{0} X_{0}} .
$$



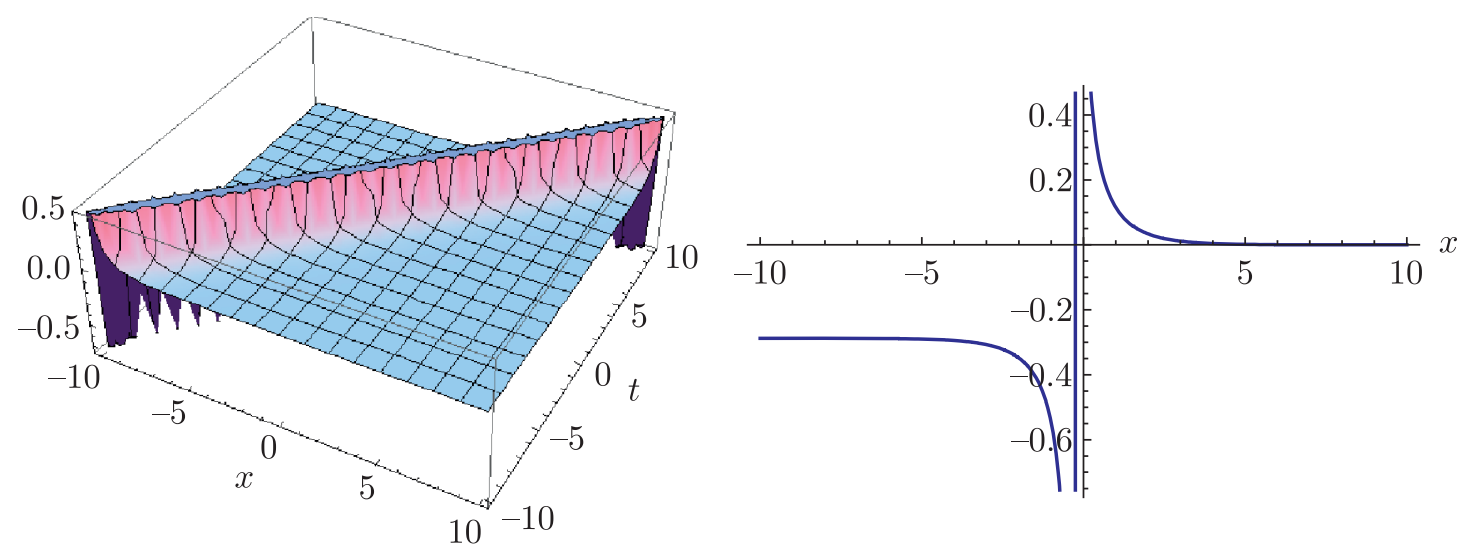

Fig. 3. The plot of Eq. (3.13) in $\mathbf{2 D}$ and $\mathbf{3 D}$ with values: $N=1, A=\frac{-1-2 C_{0}+\sqrt{1+1174 C_{0}^{2}}}{9 C_{0}}$, $C_{0}=1, X_{0}=1, C_{1}=1$.
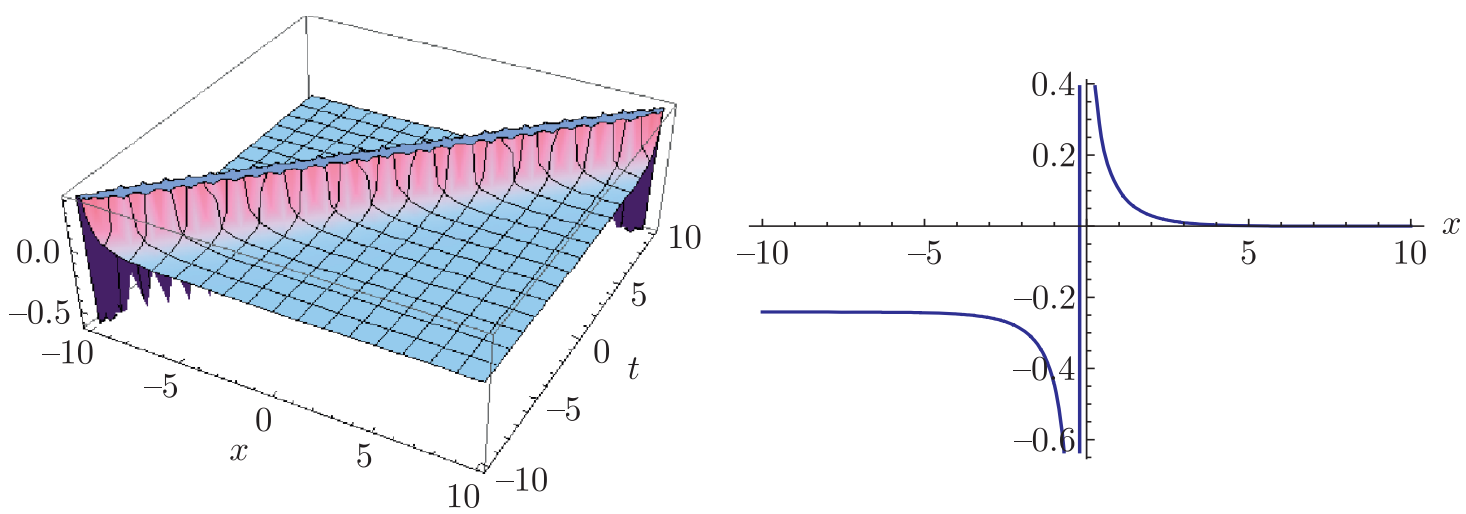

Fig. 4. The plot of Eq. (3.13) in 2D and 3D with values: $N=1, A=\frac{-1-2 C_{0}-\sqrt{1+1174 C_{0}^{2}}}{9 C_{0}}$, $C_{0}=1, X_{0}=1, C_{1}=1$.
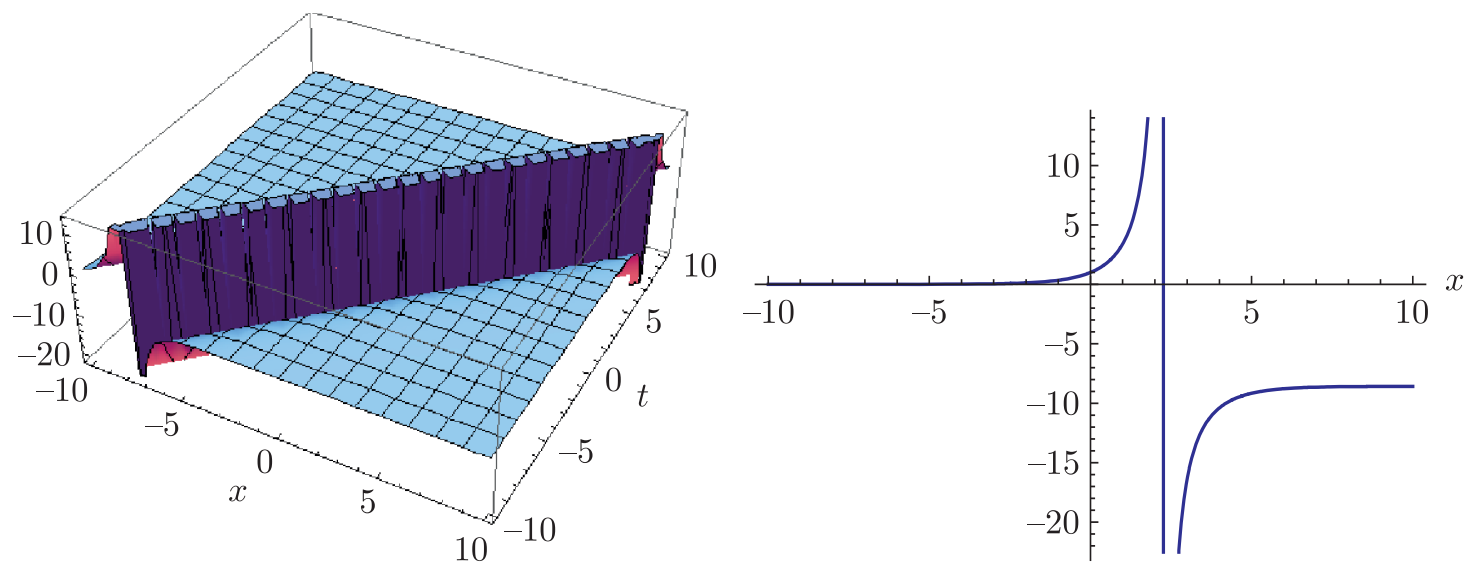

Fig. 5. The plot of Eq. (3.15) in $\mathbf{2 D}$ and $\mathbf{3 D}$ with values: $N=-1, A=\frac{2-3 C_{0}+\sqrt{9 C_{0}^{2}-102 C_{0}+4}}{6 C_{0}}$, $C_{0}=1, X_{0}=1, C_{1}=1$. 

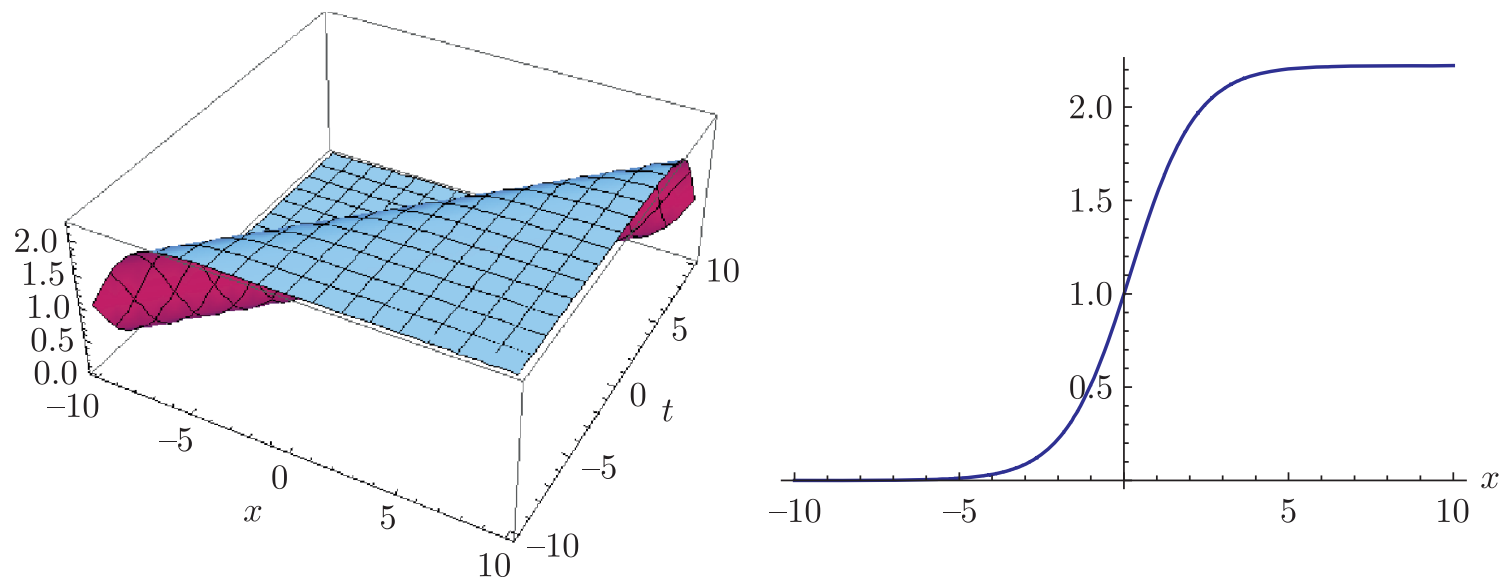

Fig. 6. The plot of Eq. (3.15) in $\mathbf{2 D}$ and $\mathbf{3 D}$ with values: $N=-1, A=\frac{2-3 C_{0}-\sqrt{9 C_{0}^{2}-102 C_{0}+4}}{6 C_{0}}$, $C_{0}=1, X_{0}=1, C_{1}=1$.

\subsection{The $(3+1)$-dimensional Kadomtsev-Petviashvili equation}

Finally, we apply the constructed approach to Eq. (1.4) mentioned above:

$$
u_{x t}+6 u_{x}^{2}+6 u u_{x x}-u_{x x x x}-u_{y y}-u_{z z}=0,
$$

Using the transformation $u(x, t)=\varphi(\zeta), \zeta=x+y+z-C_{0} t$, we get

$$
-\left(C_{0}+2\right) \varphi_{\zeta \zeta}+6\left(\varphi_{\zeta}\right)^{2}+6 \varphi \varphi_{\zeta \zeta}-\varphi_{\zeta \zeta \zeta}=0 .
$$

Integrating twice, we obtain

$$
-\left(C_{0}+2\right) \varphi+6 \varphi^{2}-\varphi_{\zeta \zeta}=0 .
$$

Again, using the transformation (3.1), substituting $\varphi_{\zeta \zeta}$ into Eq. (3.17) and equating the coefficients of different powers of $\varphi(\zeta)$ to zero gives $A=\frac{1}{N}, N= \pm \sqrt{C_{0}-2}$ :

(i) when

$$
A=\frac{1}{N}, \quad N=+\sqrt{C_{0}-2},
$$

the solution is

$$
\varphi(\zeta)=\frac{\left(C_{0}-2\right) e^{-\sqrt{C_{0}-2} \zeta}}{\left(\sqrt{C_{0}-2}-e^{-\sqrt{C_{0}-2} \zeta}\right)+\left(C_{0}-2\right) X_{0}} ;
$$

(ii) when

$$
A=\frac{1}{N}, \quad N=-\sqrt{C_{0}-2},
$$

the solution is

$$
\varphi(\zeta)=\frac{\left(2-C_{0}\right) e^{\sqrt{C_{0}-2} \zeta}}{\left(\sqrt{C_{0}-2}+e^{\sqrt{C_{0}-2} \zeta}\right)+\left(2-C_{0}\right) X_{0}} .
$$



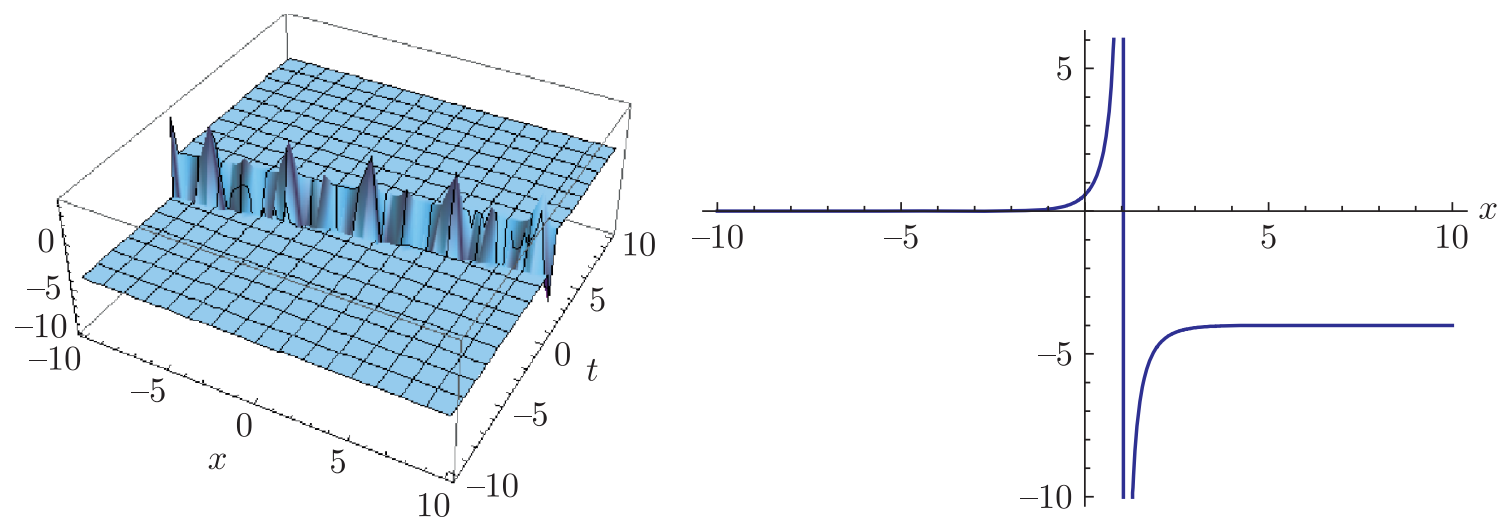

Fig. 7. The plot of Eq. (3.18) in $2 \mathrm{D}$ and $3 \mathrm{D}$ with values: $N=2, A=\frac{1}{N}, N=\sqrt{C_{0}-2}, C_{0}=6, X_{0}=1$, $C_{1}=1$.
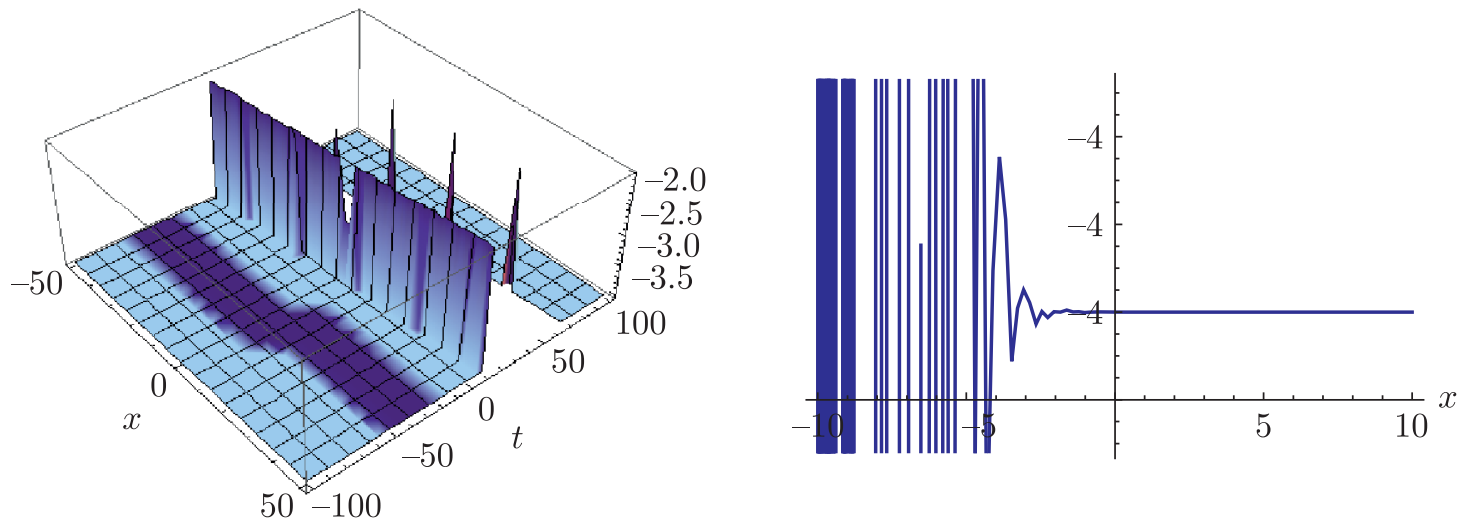

Fig. 8. The plot of Eq. (3.19) in $2 \mathrm{D}$ and $3 \mathrm{D}$ with values: $N=-2, A=\frac{1}{N}, N=-\sqrt{C_{0}-2}, C_{0}=6$, $X_{0}=1, C_{1}=1$.

\section{The variational iteration method}

Consider a differential equation with inhomogeneous term $f(\zeta)$. The linear and the nonlinear operators $R$ and $S$ become

$$
L H+N H=f(\zeta)
$$

The VIM proposes the following correction functional for Eq. (4.1):

$$
H_{m+1}(\zeta)=H_{m}(\zeta)+\int_{0}^{\zeta} \lambda(t)\left(L H_{m}(t)+N \widetilde{H}_{m}(t)-g(t)\right) d t
$$

where $\lambda$ is a general Lagrange multiplier, which can be optimally identified via the variational theory, and $\widetilde{H}_{m}$ is a restricted variation which means $\delta \widetilde{H}_{m}$. The Lagrange multiplier $\lambda$ is crucial and critical in the method, and it can be a constant or a function. With $\lambda$ determined, an iteration formula should be used for determination of the successive approximations $H_{m+1}(\zeta)$; 
$n \geqslant 0$ of the solution $H(\zeta)$. The zeros approximation $H_{0}$ can be any selective function. However, using the initial values, $H(0)$ and $H^{\prime}(0)$ are preferably used for the selective zeros approximation $u_{0}$, as will be seen later. Consequently, the solution is given by $H(\zeta)=\lim _{\zeta \rightarrow \infty} H_{m}(\zeta)$. It is interesting to point out that we formally derived the distinct forms of the Lagrange multipliers $\lambda$ in [18], hence we skip details. We only set a summary of the obtained results.

It is important to give the significant forms of Eq. (4.2) briefly according to the Lagrange multipliers in these results.

For the first-order ODE of the form

$$
H^{\prime}+q(\zeta) H=p(\zeta), \quad H(0)=\rho,
$$

we find that $\lambda=-1$, and the correction function gives the iteration formula

$$
H_{m+1}(\zeta)=H_{m}(\zeta)-\int_{0}^{\zeta}\left(H_{m}^{\prime}(t)+q(t) H_{m}(t)-p(t)\right) d t
$$

For the second-order ODE of the form

$$
\begin{gathered}
H^{\prime \prime}(\zeta)+c H^{\prime}(\zeta)+d h(\zeta)=g(\zeta), \\
H(0)=\rho, \quad H^{\prime}(0)=\eta,
\end{gathered}
$$

we find that $\lambda=t-x$, and the correction function gives the iteration formula

$$
H_{m+1}(\zeta)=H_{m}(\zeta)+\int_{0}^{\zeta}(t-x)\left(H_{m}^{\prime \prime}(t)+c H_{m}^{\prime}(t)+d H_{m}-g(t)\right) d t
$$

For the third-order ODE of the form

$$
\begin{gathered}
H^{\prime \prime \prime}(\zeta)+c H^{\prime \prime}(\zeta)+d H^{\prime}(\zeta)+e H(\zeta)=g(\zeta), \\
H(0)=\rho, \quad H^{\prime}(0)=\eta, \quad H^{\prime \prime}(0)=\sigma
\end{gathered}
$$

we find that $\lambda=-\frac{1}{2 !}(t-x)^{2}$, and the correction function gives the iteration formula

$$
H_{m+1}(\zeta)=H_{m}(\zeta)-\frac{1}{2 !} \int_{0}^{\zeta}(t-x)^{2}\left(H_{m}^{\prime \prime \prime}(t)+c H_{m}^{\prime \prime}(t)+d H_{m}^{\prime}(t)+e H_{m}-g(t)\right) d t
$$

Consequently, for the general form of ODE

$$
\begin{gathered}
H^{(m)}+f\left(H^{\prime}, H^{\prime \prime}, H^{\prime \prime \prime} \ldots, H^{(m-1)}\right)=g(\zeta), \\
H(0)=\rho_{0}, \quad H^{\prime}(0)=\rho_{1}, \quad H^{\prime \prime}(0)=\rho_{2} \ldots, H^{m-1}(0)=\rho_{m-1} .
\end{gathered}
$$

The Lagrange multiplier $\lambda$ takes the general form $\lambda=\frac{(-1)^{m}}{(m-1) !}(t-x)^{m-1}$, while the general form of iteration rule becomes

$$
H_{m+1}(\zeta)=H_{m}(\zeta)+\frac{(-1)^{m}}{(m-1) !} \int_{0}^{\zeta}(t-x)^{m-1}\left(H^{(m)}+f\left(H^{\prime}, H^{\prime \prime}, H^{\prime \prime \prime}, \ldots, H^{(m-1)}\right)-g(t)\right) d t
$$


Furthermore, the zero approximation $H_{0}(\zeta)$ can be perfectly selected to be

$$
H_{0}(\zeta)=H_{0}(0)+H^{\prime}(0) \zeta+\frac{1}{2 !} H^{\prime \prime}(0) \zeta^{2}+\frac{1}{3 !} H^{\prime \prime \prime}(0) \zeta^{3} \ldots+\frac{1}{(m-1) !} H^{m-1}(0) \zeta^{m-1},
$$

where $m$ is the order of the ODE.

\subsection{The Camassa-Holm equation}

For the third-order differential equation (3.2) mentioned above we have

$$
-C_{0} \varphi_{\zeta}+C_{0} \varphi_{\zeta \zeta \zeta}+3 \varphi \varphi_{\zeta}-2 \varphi_{\zeta} \varphi_{\zeta \zeta}-\varphi \varphi_{\zeta \zeta \zeta}=0 .
$$

Case 1: Under the initial condition

$$
A=\frac{25}{16}, \quad N=1, \quad \varphi(0)=16, \quad \varphi^{\prime}(0)=-400,
$$

according to the variational iteration method, the first and second iterations at $\varphi(0)=16$ and $\varphi^{\prime}(0)=-400$ for each of the following cases can be introduced as

$$
\begin{gathered}
\varphi_{0}(\zeta)=\varphi(0)+\zeta \varphi^{\prime}(0), \quad \varphi_{0}(\zeta)=16-400 \zeta, \\
\varphi_{1}(\zeta)=\varphi_{0}(\zeta)-\int_{0}^{\zeta}\left(-\varphi_{0}^{\prime}(t)+\varphi_{0}^{\prime \prime \prime}(t)+3 \varphi_{0}(t) \varphi_{0}^{\prime}(t)-2 \varphi_{0}^{\prime}(t) \varphi_{0}^{\prime \prime}(t)-\varphi_{0}(t) \varphi_{0}^{\prime \prime \prime}(t)\right) d t \\
\varphi_{1}=16-400 \zeta-\int_{0}^{\zeta}\left[400-1200(16-400 t] d t=16+18400 \zeta-240000 \zeta^{2},\right. \\
\varphi_{2}(\zeta)=\varphi_{1}(\zeta)-\int_{0}^{\zeta}\left(-\varphi_{1}^{\prime}(t)+\varphi_{1}^{\prime \prime \prime}(t)+3 \varphi_{1}(t) \varphi_{1}^{\prime}(t)-2 \varphi_{1}(t) \varphi_{1}^{\prime \prime}(t)-\varphi_{1}(t) \varphi_{1}^{\prime \prime \prime}(t)\right) d t \\
\varphi_{2}(\zeta)=16-884336 \zeta-519763976 \zeta^{2}+13558729530 \zeta^{3}-86544060000 \zeta^{4} .
\end{gathered}
$$
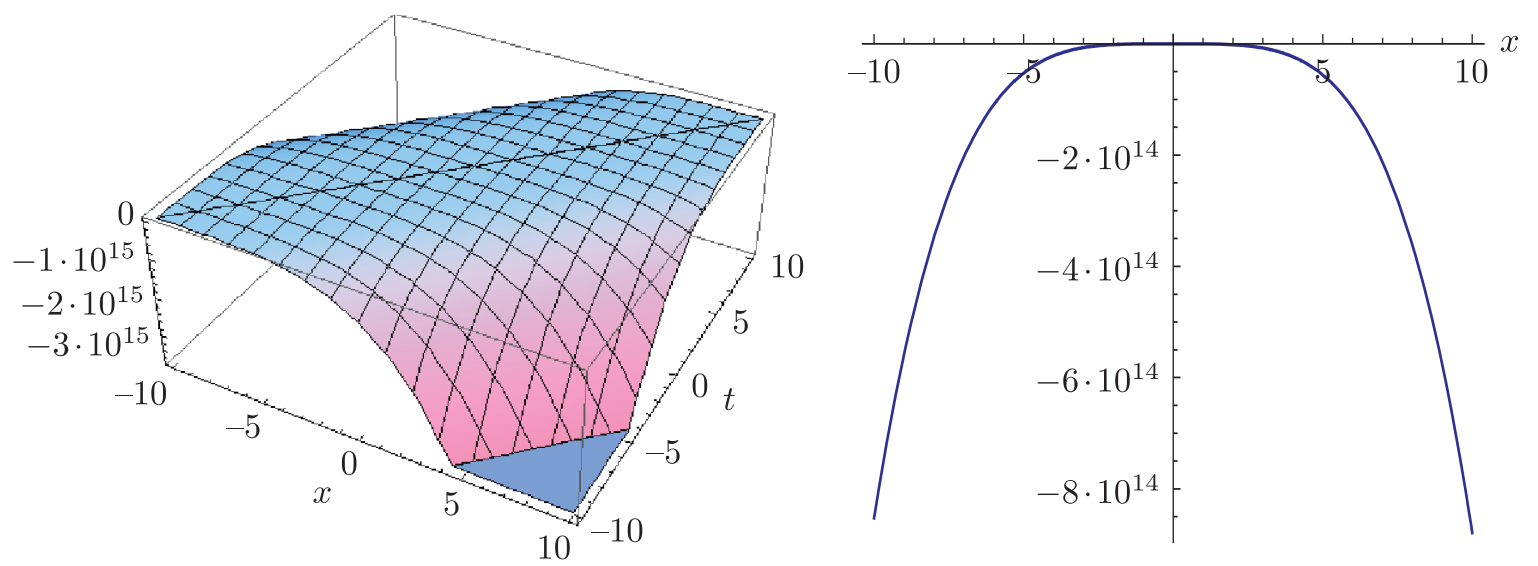

Fig. 9. The plot of Eq. (4.14) in $2 \mathrm{D}$ and $3 \mathrm{D}$ with values: $N=1, A=\frac{25}{16 C_{0}}, C_{0}=1, X_{0}=1, C_{1}=1$. 
Case 2: Under the initial condition $A=\frac{-1}{2}, N=-1, \varphi(0)=-1, \varphi^{\prime}(0)=0.5$,

$$
\begin{gathered}
\varphi_{0}(\zeta)=\varphi(0)+\zeta \varphi^{\prime}(0), \quad \varphi_{0}(\zeta)=1+0.5 \zeta, \\
\varphi_{1}(\zeta)=\varphi_{0}(\zeta)-\int_{0}^{\zeta}\left(-\varphi_{0}^{\prime}(t)+\varphi_{0}^{\prime \prime \prime}(t)+3 \varphi_{0}(t) \varphi_{0}^{\prime}(t)-2 \varphi_{0}^{\prime}(t) \varphi_{0}^{\prime \prime}(t)-\varphi_{0}(t) \varphi_{0}^{\prime \prime \prime}(t)\right) d t \\
\varphi_{1}=-1+0.5 \zeta-\int_{0}^{\zeta}\left[-0.5+3(-1+0.5 t] d t=-1+4 \zeta-\frac{7}{8} \zeta^{2},\right. \\
\varphi_{2}(\zeta)=\varphi_{1}(\zeta)-\int_{0}^{\zeta}\left(-\varphi_{1}^{\prime}(t)+\varphi_{1}^{\prime \prime \prime}(t)+3 \varphi_{1}(t) \varphi_{1}^{\prime}(t)-2 \varphi_{1}(t) \varphi_{1}^{\prime \prime}(t)-\varphi_{1}(t) \varphi_{1}^{\prime \prime \prime}(t)\right) d t, \\
\varphi_{2}(\zeta)=-1+18.5 \zeta-32.6 \zeta^{2}+7.7 \zeta^{3}-1.2 \zeta^{4} .
\end{gathered}
$$
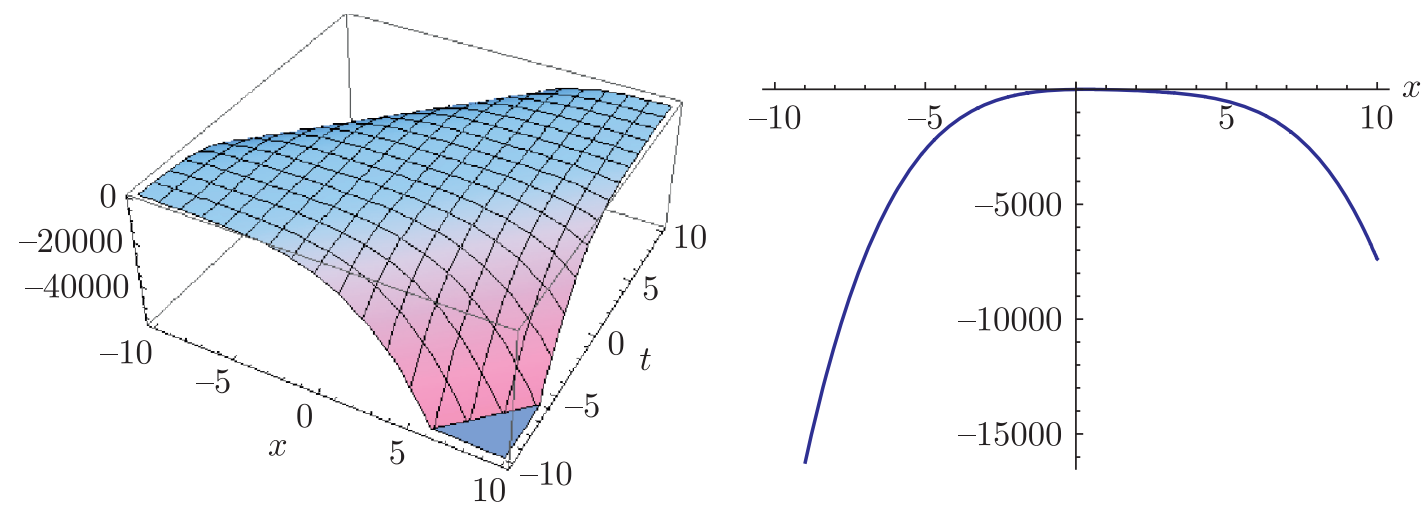

Fig. 10. The plot of Eq. (4.16) in $2 \mathrm{D}$ and $3 \mathbf{D}$ with values: $N=-1, A=-0.5$.

\subsection{The Novikov - Veselov equation}

For the third-order differential equation (3.11) mentioned above we have

$$
-C_{0} \varphi_{\zeta}+C_{0} \varphi_{\zeta \zeta \zeta}+4 \varphi \varphi_{\zeta}-2 \varphi \varphi_{\zeta} \varphi_{\zeta \zeta}-\varphi^{2} \varphi_{\zeta \zeta \zeta}=0 .
$$

Case 1: Under the initial condition

$$
A=3.5, \quad N=1, \quad \varphi(0)=1, \quad \varphi^{\prime}(0)=-4.5,
$$

according to the variational iteration method, the first and second iterations at $\varphi(0)=1$, $\varphi^{\prime}(0)=-4.5$ for each of the following cases can be introduced as

$$
\begin{gathered}
\varphi_{0}(\zeta)=\varphi(0)+\zeta \varphi^{\prime}(0), \quad \varphi_{0}(\zeta)=1-4.5 \zeta \\
\varphi_{1}(\zeta)=\varphi_{0}(\zeta)-\int_{0}^{\zeta}\left(-\varphi_{0}^{\prime}(t)+\varphi_{0}^{\prime \prime \prime}(t)+4 \varphi_{0}(t) \varphi_{0}^{\prime}(t)-2 \varphi(t) \varphi_{0}^{\prime}(t) \varphi_{0}^{\prime \prime}(t)-\varphi_{0}^{2}(t)(t) \varphi_{0}^{\prime \prime \prime}(t)\right) d t
\end{gathered}
$$



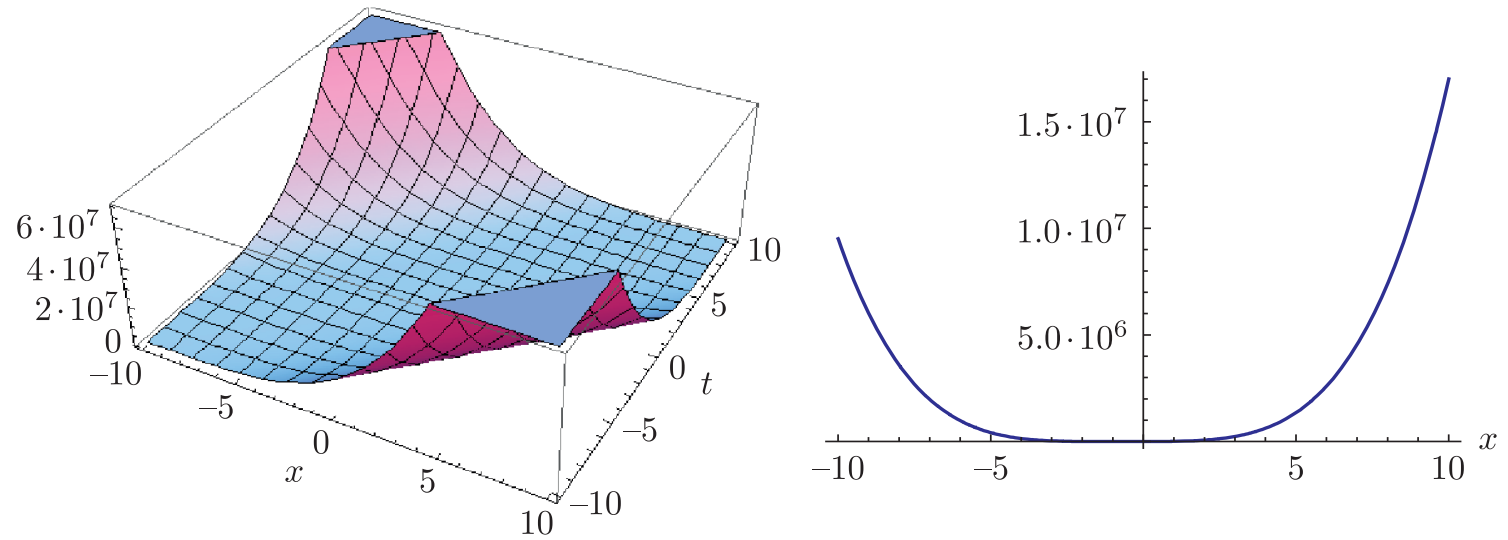

Fig. 11. The plot of Eq. (4.19) in $\mathbf{2 D}$ and $\mathbf{3 D}$ with values: $N=1, A=3.5, C_{0}=1, X_{0}=1, C_{1}=1$.

$$
\begin{gathered}
\varphi_{1}=1-4.5 \zeta-\int_{0}^{\zeta}[4.5+4(1-4.5 t)] d t=1-13 \zeta+9 \zeta^{2} \\
\varphi_{2}(\zeta)=\varphi_{1}(\zeta)-\int_{0}^{\zeta}\left(-\varphi_{1}^{\prime}(t)+\varphi_{1}^{\prime \prime \prime}(t)+4 \varphi_{1}(t) \varphi_{1}^{\prime}(t)-2 \varphi(t) \varphi_{1}^{\prime}(t) \varphi_{1}^{\prime \prime}(t)-\varphi_{1}^{2}(t)(t) \varphi_{1}^{\prime \prime \prime}(t)\right) d t \\
\varphi_{2}(\zeta)=1-442 \zeta+3042.4 \zeta^{2}-3744 \zeta^{3}+1296 \zeta^{4} .
\end{gathered}
$$

Case 2: Under the initial condition

$$
A=-4, \quad N=1, \quad \varphi(0)=1, \quad \varphi^{\prime}(0)=-3,
$$

according to the variational iteration method, the first and second iterations at $\varphi(0)=1$, $\varphi^{\prime}(0)=-3$ are

$$
\begin{gathered}
\varphi_{0}(\zeta)=\varphi(0)+\zeta \varphi^{\prime}(0), \quad \varphi_{0}(\zeta)=1-3 \zeta \\
\varphi_{1}(\zeta)=\varphi_{0}(\zeta)-\int_{0}^{\zeta}\left(-\varphi_{0}^{\prime}(t)+\varphi_{0}^{\prime \prime \prime}(t)+4 \varphi_{0}(t) \varphi_{0}^{\prime}(t)-2 \varphi(t) \varphi_{0}^{\prime}(t) \varphi_{0}^{\prime \prime}(t)-\varphi_{0}^{2}(t)(t) \varphi_{0}^{\prime \prime \prime}(t)\right) d t \\
\varphi_{1}=1-3 \zeta-\int_{0}^{\zeta}[-1+4(1-3 t)] d t=1-6 \zeta+6 \zeta^{2}, \\
\varphi_{2}(\zeta)=\varphi_{1}(\zeta)-\int_{0}^{\zeta}\left(-\varphi_{1}^{\prime}(t)+\varphi_{1}^{\prime \prime \prime}(t)+4 \varphi_{1}(t) \varphi_{1}^{\prime}(t)-2 \varphi(t) \varphi_{1}^{\prime}(t) \varphi_{1}^{\prime \prime}(t)-\varphi_{1}^{2}(t)(t) \varphi_{1}^{\prime \prime \prime}(t)\right) d t \\
\varphi_{2}(\zeta)=1-125 \zeta+433 \zeta^{2}-718 \zeta^{3}+360 \zeta^{4} .
\end{gathered}
$$

\subsection{The $(3+1)$-dimensional Kadomtsev-Petviashvili equation}

For the second-order differential equation (3.17) mentioned above we have

$$
-8 \varphi+6 \varphi^{2}-\varphi_{\zeta \zeta}=0 .
$$



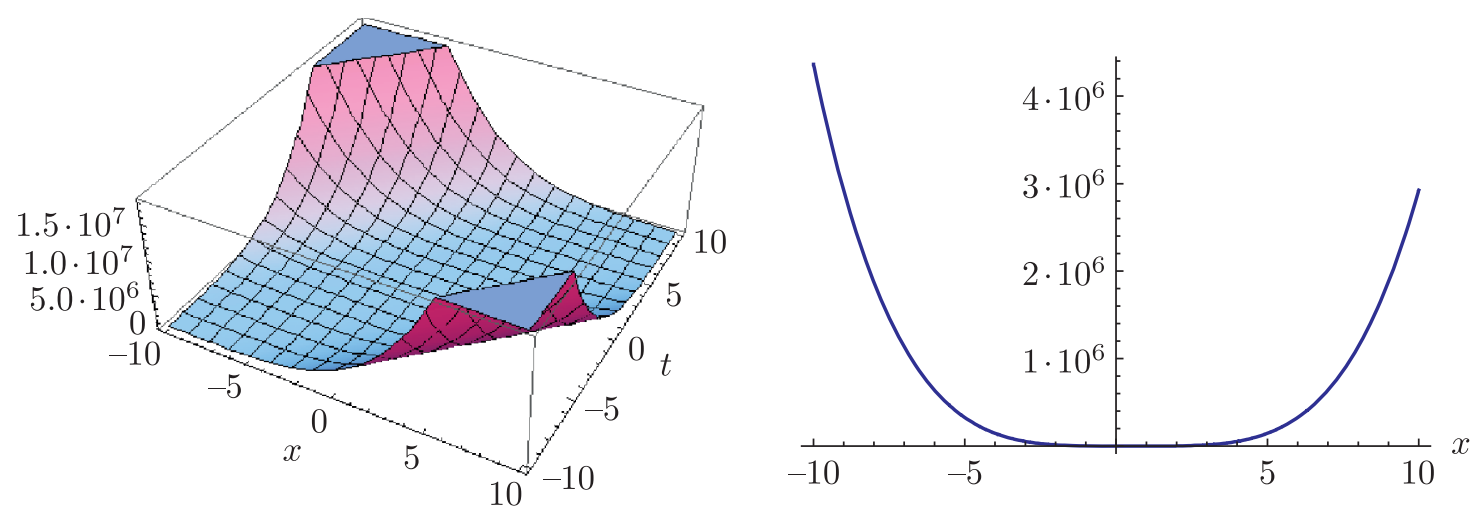

Fig. 12. The plot of Eq. (4.22) in 2D and 3D with values: $N=-1, A=-4, C_{0}=1, X_{0}=1, C_{1}=1$.

Case 1: Under the initial condition

$$
A=0.5, \quad N=2, \quad \varphi(0)=1, \quad \varphi^{\prime}(0)=-3,
$$

according to the variational iteration method, the first and second iterations at $\varphi(0)=1$, $\varphi^{\prime}(0)=-3$ are

$$
\begin{gathered}
\varphi_{0}(\zeta)=\varphi(0)+\zeta \varphi^{\prime}(0), \quad \varphi_{0}(\zeta)=1-3 \zeta, \\
\varphi_{1}(\zeta)=\varphi_{0}(\zeta)-\int_{0}^{\zeta}\left(-8 \varphi_{0}(t)+6 \varphi_{0}^{2}(t)-\varphi_{0}^{\prime \prime}(t)\right) d t \\
\varphi_{1}=1-3 \zeta-\int_{0}^{\zeta}\left[-8(1-3 \zeta)+6(1-3 \zeta)^{2}\right] d t=1-\zeta+6 \zeta^{2}-18 \zeta^{3}, \\
\varphi_{2}(\zeta)=\varphi_{1}(\zeta)-\int_{0}^{\zeta}\left(-8 \varphi_{1}(t)+6 \varphi_{1}^{2}(t)-\varphi_{1}^{\prime \prime}(t)\right) d t, \\
\varphi_{2}(\zeta)=-1+16 \zeta-106 \zeta^{2}-3 \zeta^{3}+36 \zeta^{4}-48.4 \zeta^{5}+216 \zeta^{6} .
\end{gathered}
$$
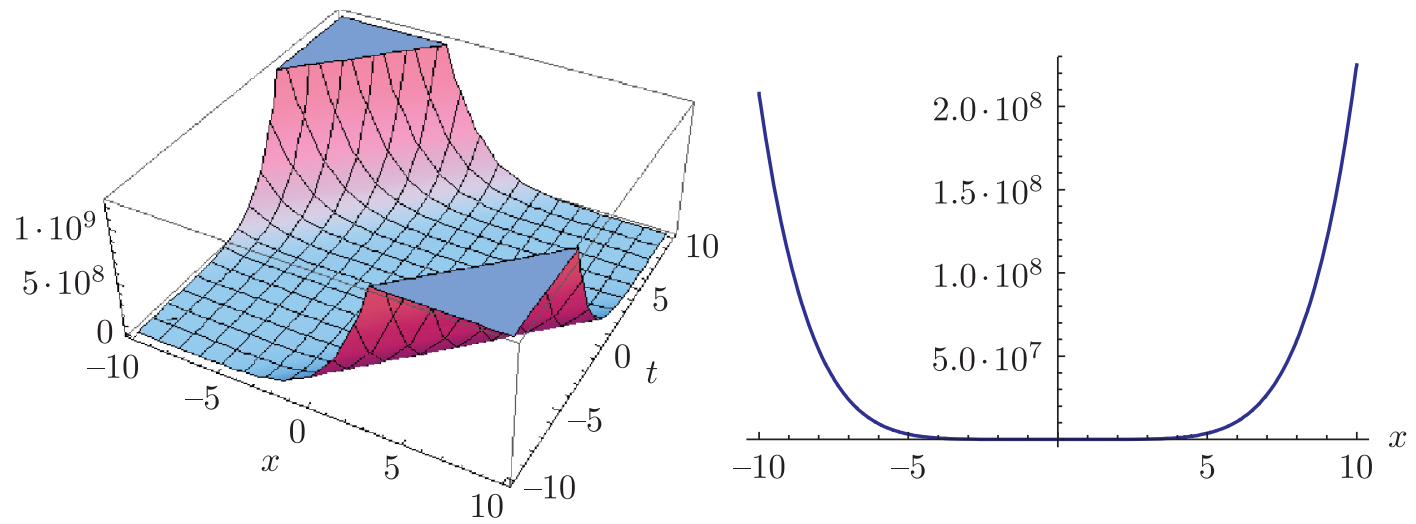

Fig. 13. The plot of Eq. (4.25) in $\mathbf{2 D}$ and $\mathbf{3 D}$ with values: $N=2, A=0.5, C_{0}=6, X_{0}=1, C_{1}=1$. 
Case 2: Under the initial condition

$$
A=-0.5, \quad N=-2, \quad \varphi(0)=1, \quad \varphi^{\prime}(0)=-1,
$$

according to the variational iteration method, the first and second iterations at $\varphi(0)=1$, $\varphi^{\prime}(0)=1$ are

$$
\begin{gathered}
\varphi_{0}(\zeta)=\varphi(0)+\zeta \varphi^{\prime}(0), \quad \varphi_{0}(\zeta)=1+\zeta, \\
\varphi_{1}(\zeta)=\varphi_{0}(\zeta)-\int_{0}^{\zeta}\left(-8 \varphi_{0}(t)+6 \varphi_{0}^{2}(t)-\varphi_{0}^{\prime \prime}(t)\right) d t, \\
\varphi_{1}=1+\zeta-\int_{0}^{\zeta}\left[-8(1+\zeta)+6(1+\zeta)^{2}\right] d t=1+3 \zeta-2 \zeta^{2}-2 \zeta^{3}, \\
\varphi_{2}(\zeta)=\varphi_{1}(\zeta)-\int_{0}^{\zeta}\left(-8 \varphi_{1}(t)+6 \varphi_{1}^{2}(t)-\varphi_{1}^{\prime \prime}(t)\right) d t, \\
\varphi_{2}(\zeta)=1+9 \zeta-2 \zeta^{2}-17.3 \zeta^{3}+20 \zeta^{4}+9.6 \zeta^{5}-8 \zeta^{6}-3.4 \zeta^{7} .
\end{gathered}
$$
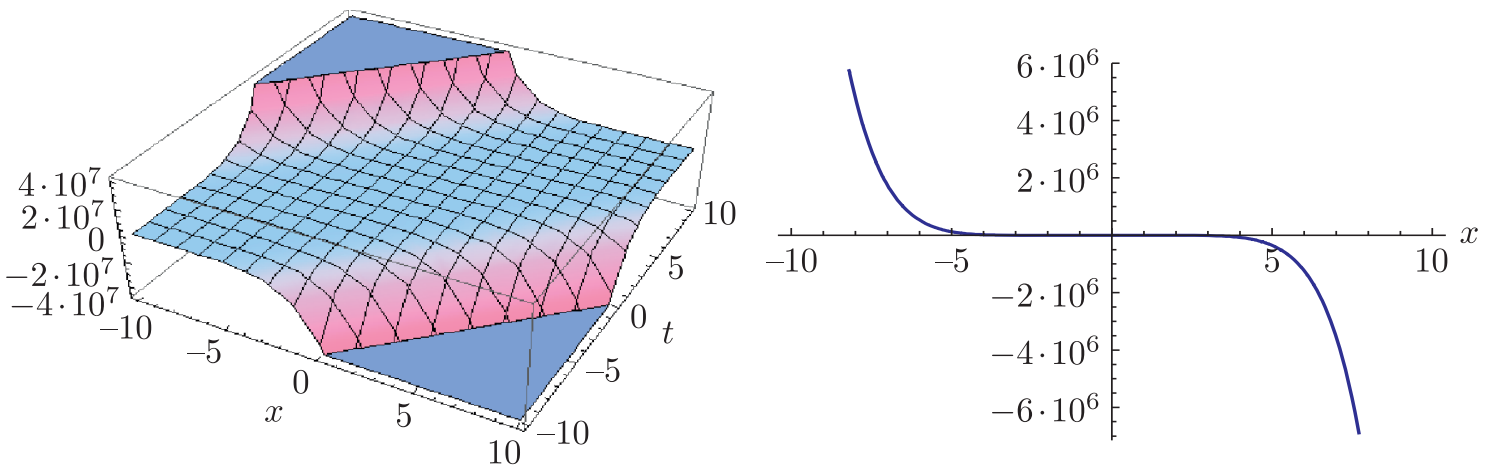

Fig. 14. The plot of Eq. (4.28) in 2D and 3D with values: $N=-2, A=-0.5, C_{0}=6, X_{0}=1, C_{1}=1$.

And for all previous cases the higher iteration can be demonstrated according to the following steps:

$$
\begin{aligned}
& \varphi_{3}(\zeta)=\varphi_{2}(\zeta)-\int_{0}^{\zeta}\left(-\varphi_{2}^{\prime}(t)+\varphi_{2}^{\prime \prime \prime}(t)+3 \varphi_{2}(t) \varphi_{2}^{\prime}(t)-2 \varphi_{2}^{\prime}(t) \varphi_{2}^{\prime \prime}(t)-\varphi_{2}(t) \varphi_{2}^{\prime \prime \prime}(t)\right) d t \\
& \varphi_{N+1}(\zeta)=\varphi_{N}(\zeta)-\int_{0}^{\xi}\left(-\varphi_{N}^{\prime}(t)+\varphi_{N}^{\prime \prime \prime}(t)+3 \varphi_{N}(t) \varphi_{N}^{\prime}(t)-2 \varphi_{N}^{\prime}(t) \varphi_{N}^{\prime \prime}(t)-\varphi_{N}(t) \varphi_{N}^{\prime \prime \prime}(t)\right) d t,
\end{aligned}
$$

using the fact that the exact solution is obtained by using $\varphi(\zeta)=\lim _{\zeta \rightarrow \infty} \varphi_{N}(\zeta)$. 


\section{References}

[1] Fuchssteiner, B. and Fokas, A.S., Symplectic Structures, Their Bäcklund Transformations and Hereditary Symmetries, Phys. D, 1981/82, vol. 4, no. 1, pp. 47-66.

[2] Constantin, A. and Escher, J., Wave Breaking for Nonlinear Nonlocal Shallow Water Equations, Acta Math., 1998, vol. 181, no. 2, pp. 229-243.

[3] Camassa, R. and Holm, D. D., An Integrable Shallow Water Equation with Peaked Solitons, Phys. Rev. Lett., 1993, vol. 71, no. 11, pp. 1661-1664.

[4] Camassa, R., Holm, D. D., and Hyman, J.M., A New Integrable Shallow Water Equation, Adv. Appl. Mech., 1994, vol.31, pp.1-33.

[5] Parkes, E. J. and Vakhnenko, V. O., Explicit Solutions of the Camassa-Holm Equation, Chaos Solitons Fractals, 2005, vol. 26, no. 5, pp. 1309-1316.

[6] Eckhordt, J., Kostenko, A., and Tesch, G., The Camassa-Holm Equation and the String Density Problem, Intenat. Math. Nachrichten, 2016, No. 233, pp.1-24.

[7] Crisan, D. and Holm, D. D., Wave Breaking for the Stochastic Camassa-Holm Equation, Phys. D, 2018, vols. 376/377, pp. 138-143.

[8] Hone, A. N.W., Lundmark, H., and Szmigielski, J., Explicit Multipeakon Solutions of Novikov's Cubically Nonlinear Integrable Camassa-Holm Type Equation, Dyn. Partial Differ. Equ., 2009, vol. 6, no. 3, pp. 253-289.

[9] Ni, L. and Zhou, Y., Well-Posedness and Persistence Properties for the Novikov Equation, J. Differential Equations, 2011, vol. 250, no. 7, pp. 3002-3021.

[10] Wu, X. and Yin, Zh., Global Weak Solutions for the Novikov Equation, J. Phys. A, 2011, vol.44, no. $5,055202,17 \mathrm{pp}$.

[11] Wu, X. and Yin, Zh., A Note on the Cauchy Problem of the Novikov Equation, Appl. Anal., 2013, vol. 92, no. 6, pp. 1116-1137.

[12] Novikov, V., Generalizations of the Camassa-Holm Equation, J. Phys. A, 2009, vol.42, no.34, 342002, $14 \mathrm{pp}$.

[13] Wu, X. and Yin, Zh., Global Weak Solutions for the Novikov Equation, J. Phys. A, 2011, vol.44, no. 5, 055202, $17 \mathrm{pp}$.

[14] Tiğlay, F., The Periodic Cauchy Problem for Novikov's Equation, Int. Math. Res. Not. IMRN, 2011, no. 20 , pp. $4633-4648$.

[15] Himonas, A. A. and Holliman, C., The Cauchy Problem for the Novikov Equation, Nonlinearity, 2012, vol. 25, no. 2, pp. 449-479.

[16] Chen, Y., Yan, Zh., and Zhang, H., New Explicit Solitary Wave Solutions for $(2+1)$-Dimensional Boussinesq Equation and (3+1)-Dimensional KP Equation, Phys. Lett. A, 2003, vol. 307, nos. 2-3, pp. 107-113.

[17] Kudryashov, N. A., The Painlevé Approach for Finding Solitary Wave Solutions of Nonlinear NonIntegrable Differential Equations, Optik, 2019, vol. 183, pp. 642-649.

[18] Wazwaz, A.-M., The Variational Iteration Method for Analytic Treatment of Linear and Nonlinear ODEs, Appl. Math. Comput., 2009, vol. 212, no.1, pp.120-134. 